\title{
Real Time Relativity: Exploratory learning of special relativity
}

\author{
C. M. Savage, a) A. Searle, and L. McCalman \\ Department of Physics, Australian National University, ACT 0200, Australia
}

(Received 18 January 2007; accepted 4 May 2007)

\begin{abstract}
"Real Time Relativity" is a computer program that lets users move at relativistic speeds through a simulated world populated with planets, clocks, and buildings. The counterintuitive and spectacular optical effects of relativity are prominent, while systematic exploration of the simulation allows the user to discover relativistic effects such as length contraction and the relativity of simultaneity. We report on the physics and technology underpinning the simulation, and our experience using it for teaching special relativity to first year students. () 2007 American Association of Physics Teachers.
\end{abstract}

[DOI: 10.1119/1.2744048]

\section{INTRODUCTION}

"Real Time Relativity"1,2 is a first person point of view gamelike computer simulation of a special relativistic world, which allows the user to move in three dimensions among familiar objects (see Fig. 1). In a first year university physics course it has proved complementary to other relativity instruction.

Because there is little opportunity for students to directly experience relativistic effects, it is often perceived as abstract, and students may find it difficult to form an integrated relativistic world-view. They find relativity interesting and exciting, but may be left bemused by the chasm between the theory and their everyday experience. ${ }^{3-5}$ Real Time Relativity can help bridge this chasm by making visual observations the basis from which the theory is deduced.

In his original relativity paper Einstein discarded personal observers, who collect information from what they see, in favor of more abstract inertial observers who use distributed arrays of rulers and conventionally synchronized clocks. ${ }^{6}$ Komar $^{7}$ and others ${ }^{8,9}$ have shown that special relativity can be formulated in terms of postulates about a personal observer's visual observations. This approach to relativity underpins our use of the Real Time Relativity simulation. It is important to distinguish between this approach based on "visual observation" and the conventional approach based on operationally defined measurements. ${ }^{10}$

Studies have shown that students may fail to learn fundamental concepts, such as the relativity of simultaneity, even after advanced instruction. ${ }^{3-5}$ This failure is due to the fact that special relativity contradicts some deeply held ideas about space and time. To overcome their nonrelativistic preconceptions students must first recognize them, and then confront them. ${ }^{4}$ The Real Time Relativity simulation can aid this process.

In the next section we discuss some relevant physics education research, before outlining the relativistic optics required to understand the simulation in Sec. III. Section IV briefly overviews the computer technology that is making interactive simulations of realistic physics, such as Real Time Relativity, increasingly practical. The students' experience of the simulation is described in Sec. V, and Sec. VI shows how the simulation provides fresh perspectives on physics such as the relativity of simultaneity. Section VII reports our evaluation of its use in a first year physics laboratory.

\section{EDUCATIONAL BACKGROUND}

There is substantial evidence for the value of active learning. ${ }^{11,12}$ Effective learning is stimulated by students participating in the construction and application of physics based world-views. ${ }^{13}$ A common factor in active learning is the cycle of developing, testing, and correcting understanding in a collaborative environment. Peer instruction is one way for active learning to occur in the classroom, ${ }^{14}$ while in the laboratory inquiry based approaches are known to be effective. ${ }^{13}$

Computer simulations can promote active learning in physics, especially where real laboratories are difficult to provide. However, a testing and development cycle is required to ensure good learning outcomes. ${ }^{15,16}$ The effectiveness of simulations is reduced by poor interfaces, ${ }^{15}$ and by students' lack of the skills required to learn from them. ${ }^{17}$ They also lose effectiveness if the exploration is not conducted according to the scientific method. ${ }^{18}$ When such issues are addressed the results can be spectacular. ${ }^{16}$

Computer simulations are most effective when directed toward clear goals, with an understanding of their strengths and limitations. ${ }^{16,18}$ They can provide an additional active learning mode, and address broad goals such as "thinking like a physicist." ${ }^{19}$ However, learning to use software increases cognitive load, lessening the capacity to learn other new material. ${ }^{17}$ The value for physics teaching of first person simulations, such as Real Time Relativity, is largely unexplored, as existing research has been on simulations of models, such as might be used by an expert physicist, rather than immersive first person simulations. ${ }^{5-18}$

Earlier software, such as Taylor's "Spacetime," used symbolic representations of objects and space-time diagrams to actively engage students with relativistic physics. ${ }^{20}$ Real Time Relativity differs from this and other physics simulations by providing a realistic, immersive, three-dimensional environment for exploration. In the context of a first year university physics class, we ask the question: Can aspects of special relativity be learned by exploration of the Real Time Relativity virtual world? Many students are comfortable interactively discovering the rules of virtual worlds; can they use this experience for discovering the rules of physics?

Successful learning from simulations is more likely if students are suitably prepared and guided. ${ }^{13,15,17}$ Preparation should develop a basic understanding of the physics which determines what is seen in the simulation. In our case this preparation includes the finite speed of light, the Doppler effect, and relativistic optical aberration. This preparation 


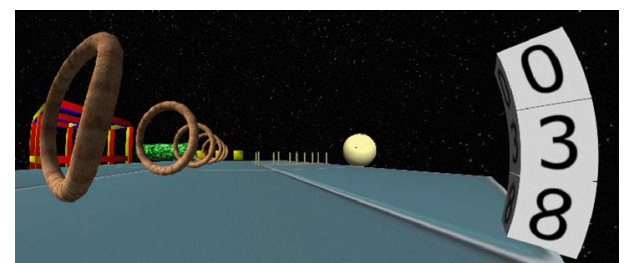

Fig. 1. The speed of the camera relative to the objects is $v=0.9682 c$. The Doppler and headlight effects have been turned off.

might use conventional interactive multimedia. ${ }^{21}$ Preparation should also include how the scientific method is used to develop understanding of novel phenomena. Because Real Time Relativity is based on visual observation, whereas the conventional approach to relativity is based on operationally defined measurements, it is important that students be able to make the connection between them. An example would be the fact that length contraction is not usually visually apparent, but an appropriate measurement would reveal it (see Sec. III). Here, as in much of modern physics, the nature of measurement is not obvious and requires explicit treatment.

Scherr, Shaffer, and Vokos have found that students' understanding of time in special relativity is poor. ${ }^{3,4}$ They conclude that "...many students who study special relativity at the undergraduate to graduate levels fail to develop a functional understanding." They identify the reason for this failure as students' misunderstanding of fundamental ideas such as the "time of an event, simultaneity, and reference frame." They have developed instructional materials to address these problems. Mermin ${ }^{22}$ has also noted that traditional relativistic pedagogy may make incorrect assumptions about students' prior knowledge. Real Time Relativity can address these problems, because fundamental ideas, such as the time of an event, have intuitive operational meanings.

\section{RELATIVISTIC OPTICS}

Some of the basic physics of relativistic optics, namely the Doppler effect and aberration, was discussed by Einstein in his first relativity paper. ${ }^{6}$ It was not until about 1960 that the pioneering work of Penrose, ${ }^{23}$ Terrell, $^{24}$ and Weisskopf ${ }^{25}$ showed that relativity gives a rich and unexpected visual environment.

In this section we summarize relativistic optics using fourvectors, because that is how it is implemented in the Real Time Relativity program (see Sec. IV). Rindler ${ }^{26}$ provides a more complete introduction, both with and without using four-vectors.

A plane light wave is described by its four-frequency $F$, which has components ${ }^{26}$

$$
F=f[1, \mathbf{n}] \text {, }
$$

where $f$ is the frequency and $\mathbf{n}=\left(n_{x}, n_{y}, n_{z}\right)$ is the unit vector in the propagation direction. From the four-frequency components in a particular frame, the components in any other frame may be found using a Lorentz transformation. The transformation between the usual standard configuration frames $S$ and $S^{\prime}$ are sufficient for our purposes. The standard configuration of frames $S$ and $S^{\prime}$ has the $S^{\prime}$ origin traveling with speed $v$ along the positive $x$ axis of $S$. The frames' origins are coincident at times $t=t^{\prime}=0$, and the corresponding coordinate axes are parallel. We will use "world" $(w)$ and "camera" (c) to refer to the frames $S$ and $S^{\prime}$,

$$
\begin{aligned}
& f_{c}=\gamma f_{w}\left(1-n_{w, x} v / c\right), \\
& f_{c} n_{c, x}=\gamma f_{w}\left(n_{w, x}-v / c\right), \\
& f_{c} n_{c, y}=f_{w} n_{w, y}, \quad f_{c} n_{c, z}=f n_{w, z},
\end{aligned}
$$

where $v$ is the relative velocity of the frames along the positive $x_{w}$ axis of the world frame and $\gamma=\left(1-v^{2} / c^{2}\right)^{-1 / 2}$. Equation (2a) expresses the Doppler effect, and the remaining equations express the dependence of the propagation direction on the relative frame velocity: an effect known as "relativistic aberration." In Real Time Relativity the objects are at rest in the world frame, and the camera frame is the user's instantaneous rest frame; the user is represented by a camera. We require the frequencies and propagation directions in the camera frame, $f_{c}$ and $\mathbf{n}_{c}$.

Because $\mathbf{n}_{c}$ is a unit vector, its $x$ component, $n_{c, x}$, is the cosine of the angle $\theta_{c}$ between the light ray and the $x_{c}$ axis: if the ray is coming toward the observer, $n_{c, x}$ changes sign. If we divide Eqs. (2b) and (2c) by Eq. (2a) and use $n_{c, x}$ $=-\cos \theta_{c}$ and $n_{w, x}=-\cos \theta_{w}$, we obtain

$$
\begin{aligned}
& \cos \theta_{c}=\frac{\cos \theta_{w}+v / c}{1+(v / c) \cos \theta_{w}}, \\
& \sin \theta_{c}=\frac{\sin \theta_{w}}{\gamma\left(1+(v / c) \cos \theta_{w}\right)} .
\end{aligned}
$$

Relativistic aberration is analogous to nonrelativistic forms of aberration that students may have experienced, for example, the dependence of the angle of falling rain on an observer's velocity, and the difference between the visual position of a high flying aircraft and that indicated by its sound. This understanding may be made quantitative using the relativistic velocity addition formulas. ${ }^{26}$

Penrose $^{23}$ showed that relativistic aberration implies that straight lines are seen as either lines or circular arcs in other frames. He also showed that a sphere, which always has a circular outline (unlike a circle, which may have an elliptical outline), will continue to have a circular outline after aberration, and hence continue to look like a sphere. These effects are immediately apparent in Real Time Relativity (see Fig. 2).

Similar observations led Terrell to argue for the "invisibility of the Lorentz contraction," 24 in favor of an interpretation of visual appearance in terms of what is now known as "Terrell rotation." It is now accepted that this interpretation is an overstatement and that Lorentz contraction is visible for small objects viewed perpendicular to the direction of relative motion. ${ }^{10}$ Such a visual observation constitutes a length measurement provided the light from the ends of the measured object is emitted simultaneously in the observer's frame. Then, if we let the (perpendicular) distance to the object be $d$ and the observed angular size be $\Delta \theta$, the object's Lorentz contracted length is $L=d \Delta \theta$ (see the Appendix).

The nonrelativistic Doppler effect may also be familiar to some students. This effect, together with the analogy to nonrelativistic aberration, emphasizes the closer relation of relativistic optics to direct experience than that of the usual space-time approach to special relativity.

A convenient form of the Doppler effect follows from Eqs. (2a) and (2b) after eliminating $n_{w, x}$ : 


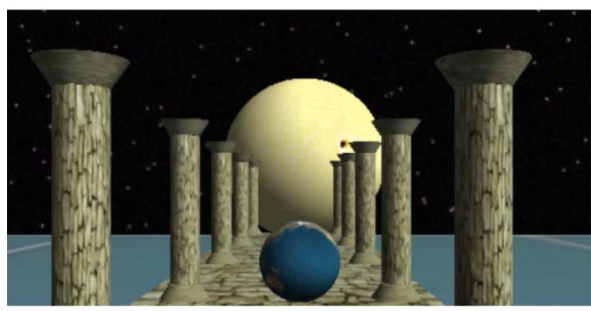

(a)

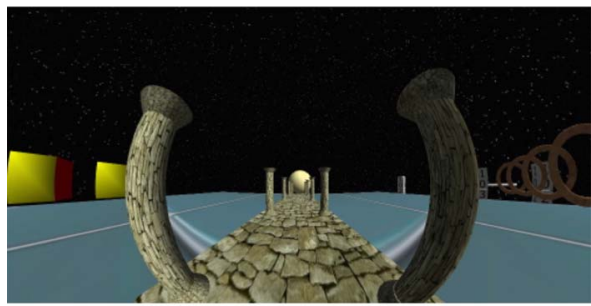

(b)

Fig. 2. (a) At rest in the world frame. (b) $v=0.9682 c$, corresponding to $\gamma$ $=4$. The Doppler and headlight effects have been turned off. In the world frame the camera is in front of its position in (a).

$$
f_{c}=\frac{\sqrt{1-v^{2} / c^{2}}}{1-(v / c) \cos \theta_{c}} f_{w}=D f_{w} .
$$

Equation (4) defines the Doppler factor $D$. For $v / c \ll 1$ the denominator is the familiar nonrelativistic wave compression or expansion. For incoming waves at $\theta_{c}=\pi / 2$ to the relative motion, the denominator is one and the observed frequency is less than the world frequency, at which they were emitted by the inverse time dilation factor $\gamma^{-1}$, which means that the period, the time between wave crests, is longer. If the wave crests are regarded as a clock, this effect is exactly time dilation.

The effect of aberration on small angles may be found by taking differentials of the inverse Lorentz transformation of the four-frequency giving 27

$$
d \theta_{c}=D^{-1} d \theta_{w} .
$$

Hence small angles transform by the inverse Doppler factor. In particular, for objects directly ahead, so that $\theta_{c}=0$, and for $v / c \ll 1$, the inverse Doppler factor is $D^{-1} \approx 1-v / c$, and objects' angular sizes are shrunk. For objects behind, $D^{-1} \approx 1$ $+v / c$, and objects are expanded. For objects perpendicular to the motion $D^{-1}=\gamma$, and as shown in the Appendix, $d \theta_{w}$ $=\gamma^{-2} L_{P} / d$, where $L_{P}$ is the object's proper length and $d$ is its perpendicular distance. Therefore the angle it subtends to the camera is $d \theta_{c}=\gamma^{-1} L_{P} / d$, from which the object's Lorentz contracted length $\gamma^{-1} L_{P}$ may be inferred.

Perhaps the most subtle of the relativistic optics effects is the headlight effect. A complete discussion was not given until 1979 by McKinley. ${ }^{28,29}$ It refers to the increased intensity of light coming from objects that are moving toward us. The intensity decreases for objects from which we are moving away. Three factors combine to produce these intensity changes: the change in the angular size of the emitting region, the Doppler change in the energy of the photons, and the change in the photon flux due to the combined effects of time dilation and the observer's motion, which is an additional manifestation of the Doppler effect. In terms of the
Doppler factor in Eq. (4) these effects contribute to the change in intensity by factors of $D^{2}, D$, and $D$, respectively, for a combined intensity change factor of $D^{4}$. For common detectors, such as the eye or a CCD camera, it is the photon number flux $P$ that is detected, and the flux changes by a factor of $D^{3}$, because the energy change per photon is irrelevant:

$$
P_{c}=D^{3} P_{w} .
$$

\section{TECHNOLOGY}

Computers can generate images incorporating special ${ }^{30}$ and general $^{31}$ relativistic optics. By the early 1990s it was possible to interactively render simple objects such as cubes. ${ }^{20,32}$ The highest quality images were generated by the ray-tracing method, which is capable of producing photorealistic images. ${ }^{33}$ However, ray-tracing is currently too slow for interactive simulations, although individual images can be strung together to make movies. ${ }^{21}$

The development of the programmable graphics processor $^{34}$ has made it possible to render complex relativistic scenes in real time. The first such systems appear to have been developed by Weiskopf ${ }^{35}$ and Bochers ${ }^{36}$ in the physics education group at the University of Tübingen. ${ }^{37}$ This group has focussed on using relativity visualization for science communication. ${ }^{38}$ Our independently developed Real Time Relativity simulation is similar to theirs, but is freely available, and is being developed as an Open Source project under the Lesser General Public License. ${ }^{1}$

The screen image displayed by Real Time Relativity is created using the computer graphics technique known as environment mapping, which renders the three-dimensional virtual world onto a two-dimensional cube map. A cube map may be visualized as the $4 \pi$ sterradian view-field mapped onto the interior surface of a cube centered on a camera, representing the user's field of view. The cube map is a data structure in which the image pixels are addressed by line of sight direction, rather than by spatial position. The relativistically correct scene is produced by transforming the cube map.

Each camera image pixel is formed by light incident from a particular direction, that is, with a specific propagation direction $\mathbf{n}_{c}$ in the camera frame. The relativistic physics problem is to find the corresponding propagation vector $\mathbf{n}_{w}$ in the world frame in which the cube map is constructed. This vector then addresses the pixel on the cube map that is mapped to the camera pixel. The resulting camera image is displayed on the screen.

A plane light wave is represented by the relativistic fourfrequency, Eq. (1). The propagation direction in the world frame is found by the inverse Lorentz transformation ${ }^{27}$ of this four-vector from the camera frame onto the world frame. This transformation is implemented as a four-dimensional matrix multiplication of the four-frequency. The transformation matrix is calculated before each frame is rendered using the current camera velocity, and is then applied to a fourfrequency constructed for each camera pixel. The transformed four-frequency has a spatial component equal to the pixel's imaging direction and the time component set to one. This spatial component addresses the cube map pixel that is then rendered to the screen.

The graphics processing units on computer video cards are designed to do four-vector matrix algebra efficiently and in 
parallel, making it possible for simulations to perform the Lorentz transformations in real time. The four-vectors that graphics processing units normally work with specify the $x$, $y$, and $z$ coordinates of a vertex and a fourth $w$ component that facilitates certain nonlinear transformations (such as translation and perspective projection), or specify the red, green, blue color and alpha (transparency) of a (texture) pixel. Because the processing of different vertices or pixels is usually independent, these operations can be performed in parallel.

The Doppler shift factor $D$ is given by the ratio of the time components of the four-frequencies in the camera and world frames [Eq. (4)]. To determine the effect of the Doppler shift on a general color requires the entire intensity spectrum. But in current graphics systems the spectrum is specified at just three frequencies: red, green, and blue. Hence interpolation is used to generate the spectrum. This simple approach, together with the lack of any infrared or ultraviolet spectra, prevents a true representation of Doppler shifted colors, and is a significant limitation of the current version of Real Time Relativity. In particular, stars do not maintain a blackbody spectrum. $28,39,40$

The headlight effect, Eq. (9), is implemented by multiplying each pixel color vector by $D^{3}$. There are significant limitations on how the resulting large intensity range is rendered to the screen by current hardware.

The graphics processing unit does the Lorentz transformations as well as its usual graphics work. First, a nonrelativistic three-dimensional scene is rendered to a cube map, then relativistic transformations are applied to it. To generate a frame, the four-frequency associated with each camera pixel is inverse Lorentz transformed to find the corresponding world frame cube map pixel. This pixel is then Doppler and intensity shifted by the graphics processing unit. An 800 $\times 600$ window has 480000 pixels, so displaying 50 frames per second requires 24 million pixel transformations per second, which is well within the capabilities of inexpensive graphics processing units. Consequently, the conventional graphics processing needed to generate the cube map limits the overall performance, not the relativistic calculations.

Real Time Relativity is programmed using Microsoft's DirectX 9 interface, so that it is independent of the details of any particular graphics processing unit. Consequently, it is only available on the Windows operating system. ${ }^{41}$ DirectX 9 includes the High Level Shader Language in which the pixel shader controlling the graphics processing unit is written.

Graphics processing units have been increasing in processing power more rapidly than central processing units. ${ }^{34}$ This increase is driven by the demand for parallel computing from the gaming community. For example, the Xbox 360 graphics processing unit has 48 32-bit processors running at $500 \mathrm{MHz}$, each capable of a floating point four-vector operation per cycle, giving nearly 100 GigaFlops, compared to a few GigaFlops for a central processing unit. ${ }^{42}$ The main limitation is that graphics processing units do data-parallel computing, in which the same operation is repeated on each element of a data array. Computational scientists are developing parallel algorithms for tasks such as solving partial differential equations. ${ }^{34}$ The Folding@Home distributed computing project has a client that runs their molecular dynamics simulations on graphics processing units, increasing computational power by about 20 times per computer. ${ }^{43}$ These devel- opments may have an impact on the kinds of physics simulations that will be possible in the future.

\section{THE REAL TIME RELATIVITY SIMULATION}

In this section we introduce Real Time Relativity as experienced by students in the first year course for physics majors at The Australian National University. ${ }^{44}$ It was used in a three hour laboratory structured to encourage exploration, while requiring that certain measurements be made and compared to theory. Students were provided with a manual with background information and both qualitative and quantitative questions. ${ }^{45}$ Many students completed the laboratory before they attended the relativistic optics lecture. Students worked in groups of two or three, and discussion was encouraged. Preparation included answering simple pre-lab questions, which were assessed at the beginning of the laboratory.

An initial problem of orientation in relativistic optics simulations arises because the speed of light is very large in everyday terms. Consequently, either the objects in the simulation must be very large, roughly light-seconds, or the speed of light must be artificially slow, as in Gamow's Mr. Tompkins story. ${ }^{46}$ In the interest of realism, we have taken the former view, which allows us to include realistic astronomical objects such as the Earth, which is 0.042 light-seconds in diameter. Figure 2(a) shows a screen from Real Time Relativity. The Earth is visible, as is the Sun behind it. ${ }^{47}$ These objects set the scale of the simulated world. Other objects, such as the columns, have been chosen for their familiar shapes, although they would be absurdly large if they existed in the real world. Familiar objects aid in the recognition of the distortions caused by relativistic aberration.

Students start by accelerating from rest down the row of columns shown in Fig. 2. At first it seems that they are moving backward. ${ }^{48}$ This experience is counterintuitive and prompts them to question what they see: the exploration has begun! The effect is due to relativistic aberration. An important way that motion is sensed is by the change in angular size as our distance to the object changes. Normally, as we approach an object its angular size increases, roughly proportionate with the distance. In contrast, the decrease in angular size due to relativistic aberration, Eq. (5), is approximately proportional to $1-(v / c) \cos \theta_{c}$, for $v / c \ll 1$, and occurs before the distance has time to change. Therefore the initial view is of objects shrinking, which is interpreted as the objects moving away, and hence as backward movement of the viewer. As they continue to accelerate, nearby objects eventually pass by, and the perception of forward motion is restored.

Colors change due to the Doppler effect Eq. (4), but the headlight effect quickly saturates the scene with bright light, dominating all other effects, due to its dependence on the third power of the Doppler factor, Eq. (6). Consequently, it is useful to be able to turn it off. Although turning it off goes against the principle of making the simulation as realistic as possible, it is difficult to see some other effects if it is left on.

The Doppler effect depends on the viewing angle, Eq. (4). There is a particular angle to the direction of motion $\theta_{0}$ for which there is no effect, because the Doppler factor $D=1$ when

$$
\cos \theta_{0}=(c / v)\left(1-\gamma^{-1}\right) .
$$

For $v=0.5 c$ this angle is $\theta_{0}=1.3\left(74^{\circ}\right)$. If students look at a pure colored object at this angle, they see a rainbow effect, 


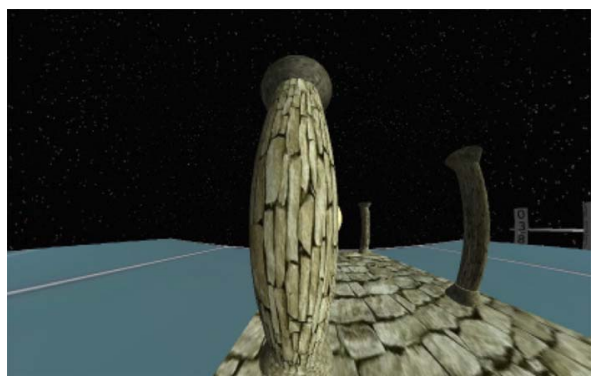

Fig. 3. Screenshot showing relativistic aberration. The camera is moving toward the center of the column with $v=0.9682 c$, so that $\gamma=4$. The Doppler and headlight effects are off.

because for directions toward the motion, $\theta<\theta_{0}$, the color is blueshifted, while the color is redshifted for directions away from the direction of motion, $\theta>\theta_{0}$.

If students already understand the nonrelativistic Doppler effect, they can be guided to discover the relativistic version. In particular, it is possible to deduce time dilation from the observation that there is reddening when viewing objects perpendicular to the direction of motion, Eq. (4).

Figure 2(b) shows the scene with the camera traveling down the row of columns with a speed corresponding to $\gamma$ $=4$. The Doppler and headlight effects have been turned off. The circular curvature of the nearest columns is due to relativistic aberration, as discussed in Sec. III. The curvature of the more distant columns is barely noticeable. However, they are shrunk by approximately the inverse Doppler factor $D^{-1}$, according to Eq. (5). The camera field of view covers a wide field in the world frame: the hoops and cubes on the edges of the image are behind the camera in the world frame.

Aberration may be understood as a consequence of the finite speed of light. The key idea is that the light that reaches the camera at a particular instant was reflected by objects at different times. The light from closer objects was reflected later than that from far away objects. This time difference is irrelevant when the camera is at rest relative to the objects, but when it is moving, the position of the objects in the camera frame depends on time. Therefore, the parts of a large object nearer the camera reflect the received light later than the further parts. If the camera is moving toward the object at a significant fraction of the speed of light, the near parts reflect when they are significantly closer and hence look bigger than the far parts, which reflected when they were further away and hence look smaller. If we are moving directly toward the middle of an object, the net result is that the middle looks fatter than the ends (see Fig. 3). If the object is off to one side, it is curved into a circular arc.

In the laboratory students are asked to measure length contraction as a function of speed, using visual observations of objects perpendicular to the camera's velocity, as discussed in Sec. III. To facilitate this measurement there is an option to draw a rectangular grid across the screen. Students are then asked to devise and perform observations of time dilation. The required conditions are similar to those required for observing length contraction: the camera must move in a direction perpendicular to the line from the camera to the clock. If they are sufficiently far apart, the distance to the clock is approximately constant, and pure time dilation is observed for a short time.

Currently, Real Time Relativity is limited to all objects

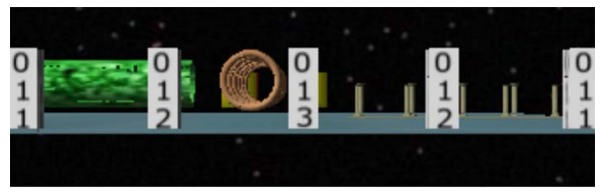

(a)

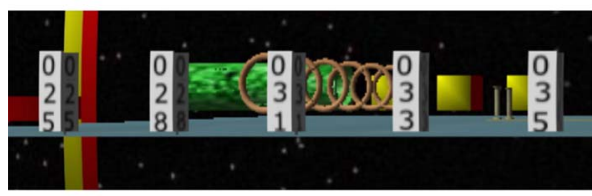

(b)

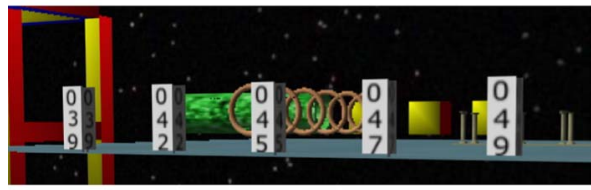

(c)

Fig. 4. Screenshots showing the relativity of simultaneity. (a) The effect of light propagation delay on the observed clocks. The camera is at rest relative to the clocks, which are lined up perpendicularly to the line of sight to the central clock. The clocks are 5 light seconds apart and read seconds. (b) The camera is moving from left to right parallel to the clocks with $v=0.5 c$. The perpendicular distance to the clocks is the same as in the top frame (about 31 light-seconds). The major contributor to the different clock readings is the relativity of simultaneity. Light delay causes clocks to the left to differ more from the central clock than those to the right. (c) The camera has been brought to rest immediately after taking the middle frame, although some time then elapsed before the image was taken. In the clocks' rest frame the different clock readings are entirely a consequence of the light propagation delay. The field of view is the same in each frame.

being at rest in the world frame. This limitation means that relativistic dynamics is not within its capabilities.

\section{THE RELATIVITY OF SIMULTANEITY}

The relativity of simultaneity is particularly difficult concept for students to learn from passive instruction. ${ }^{3-5}$ In order that students might discover the relativity of simultaneity for themselves, Real Time Relativity includes clocks in the world frame. Even when the camera is at rest in the world frame, clocks at different distances from the camera are seen to read different times due to the light propagation delay [see Fig. 4(a)]. Students generally have no difficulty recognizing and utilizing this fact. ${ }^{3-5}$ Note that clocks at the same distance from the camera read the same time.

Figure 4(b) shows the same view of the clocks, but with the camera moving with $v=0.5 c$ parallel to the clocks from left to right. The camera is looking perpendicularly to its direction of motion. Note that the eye gets confusing cues from this image, because the clocks are rotated as if we were looking at them from in front, but we are not. This effect is a result of relativistic aberration known as "Terrell rotation," which was mentioned in Sec. III. ${ }^{24}$ Because we are looking at the clocks in the direction perpendicular to the motion, length contraction by the factor $\gamma^{-1}=0.87$ is found by measuring the clock's positions in Figs. 4(b) and 4(a). The ratio of the distances between the left edges of the second and fourth clocks, measured directly from the images, is the length contraction factor, as discussed in Sec. III. 
The relativity of simultaneity is apparent from the readings on the clocks in Fig. 4(b). The right-most clock is ahead of the left-most by $10 \mathrm{~s}$. This time difference cannot be explained by light delay in the camera frame, because the observed time difference is too large, and the times increase from left to right. However, it is explained by light delay in the clocks' frame. Students can determine the light delay by immediately stopping the camera relative to the clocks. Due to relativistic aberration, they must then look back to see the clocks: this view is shown in Fig. 4(c). In the clocks' rest frame the camera is not opposite the clocks, but is to their right. From this perspective it is clear why the clocks read as they do: the left-most clock is furthest and reads earliest, while the right-most is closest and reads latest. The time difference between them is exactly that seen by the moving camera.

Let us restate the argument in terms of two photographers: Alice is moving relative to the clocks, and Bob is stationary relative to the clocks. Both Alice and Bob take photographs of the clocks at the event "Click," chosen so that Alice, in her own frame, is approximately equidistant from the locations of the clocks when they emitted the photographed light. ${ }^{49}$ Both Alice and Bob are sampling the set of photons originating from the clocks and present at Click. These photons carry the same information, in particular, the times read by the clocks when they were emitted. The different times of the different clocks is understood by Bob as a result of the light propagation delay over the different distances to the clocks. The clocks were at approximately the same distance from Alice when they emitted the light, so she requires another explanation: a new physical effect, the relativity of simultaneity. The relativity postulate ensures that what is true for these clocks is true for any clocks, and hence for time itself. A complete discussion is given in the Appendix.

\section{LABORATORY EVALUATION}

Real Time Relativity was incorporated in a first year laboratory session at The Australian National University. ${ }^{44}$ The course included nine lectures and three tutorials on special relativity. One lecture was devoted to relativistic optics.

The content of the laboratory has been discussed in Sec. V. Its effectiveness was assessed in three ways. (1) Students completed surveys before and after the laboratory. (2) One of the authors was present as an observer in each laboratory to record how students interacted with the simulation. A laboratory demonstrator was also present. (3) Students recorded their work in laboratory log books that were assessed. The course's final exam was used to check that exposure to Real Time Relativity had not reduced the students' ability to solve conventional relativistic physics problems.

The pre-laboratory surveys indicated that students usually had prior knowledge of relativity and were eager to learn more. However, they tended to perceive it as an abstract subject. The post-laboratory surveys indicated that students believed they had learned about relativity from the simulation, and that it had stimulated their interest. Some students reported that the "concrete" or "visual" nature of the simulation was helpful: ${ }^{50}$

\section{"Real Time Relativity is very useful-many people are visual learners."}

Students often reported that the laboratory manual was too prescriptive and did not allow them to adequately pursue their own investigations. This criticism focussed on the quantitative exercises:

$$
\begin{aligned}
& \text { "Why are we forcing equations from the } \\
& \text { simulation?" }
\end{aligned}
$$

There were also many complaints about the difficulty of using the program, and the inadequate time available to develop proficiency with navigation through the virtual world:

"The controls were really, really hard to use."

The laboratory observer enabled a testing and refinement cycle. We identified problems and corrected them before the next student group took the laboratory. In particular, students often tried to push simulations to the limits to see what happened, behavior noted by the University of Colorado Physics Education Technology group. ${ }^{15,16}$ If a simulation does not respond sensibly, students lose confidence in its reliability. Observers were able to monitor what engaged students and what frustrated them. The most engaging aspect was the exploration of a novel and open ended world. Among the more frustrating aspects were the simulation's controls not behaving in ways students considered natural.

The log books completed during the laboratory did not capture the excitement that was observed in working laboratory groups. However, successful quantitative measurements were generally made of the Doppler effect, time dilation, and length contraction as functions of speed.

Our experience confirmed the importance of developing educational software through a testing and refinement cycle. $^{15,16}$ Students used the simulation in ways we had not anticipated, and had ideas different than the authors about what constituted a natural user interface. The flaws in the simulation had a bigger negative impact on the students than expected. Students sometimes attributed their lack of understanding of the physics of the simulation to a "bug," even when there was none, rather than to their need to develop better understanding. 15

Our experience suggests that Real Time Relativity can stimulate discovery learning and provide complementary learning opportunities to those provided by lectures and problem solving tutorials. Realizing its full value will require further cycles of testing and development. The next time we use it, we shall require students to "play" with the simulation as part of the pre-laboratory preparation, so that they have some familiarity with the controls and with the peculiarities of navigation in a relativistic world. We shall also provide more opportunity for open ended exploration, as this aspect appears to be its strength.

\section{CONCLUSION}

Real Time Relativity is an immersive physics simulation of a kind that is becoming increasingly accessible due to the improving cost effectiveness of computer technology. It gives students the opportunity to discover and confront their misconceptions about relativity and to construct resolutions.

Our experience with Real Time Relativity suggests that it provides new perspectives on special relativity, which may be particularly valuable to students who prefer the concrete over the abstract. Important physics, such as the relativity of simultaneity, can be introduced with minimal mathematics, 


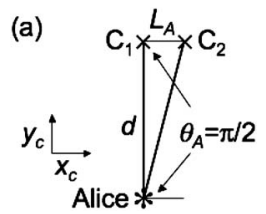

(b)

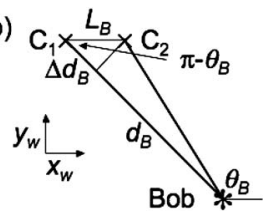

Fig. 5. Schematic diagrams for the relativity of simultaneity. Both panels refer to the time of event Click, indicated by *, when the photographs are taken. The lines from clocks $C_{1}$ and $C_{2}$ to Alice and Bob are the paths taken by the light forming the photographs in their respective frames. (a) Alice's frame. (b) Bob's frame in which the clocks are at rest.

which may broaden the group of students who can learn relativity. The educational value of first person simulations, like Real Time Relativity, is an interesting area for further physics education research.

\section{ACKNOWLEDGMENT}

The authors acknowledge the assistance of Dr. K. Wilson with the implementation and evaluation of the Real Time Relativity laboratory.

\section{APPENDIX: THE RELATIVITY OF SIMULTANEITY}

In this appendix we expand on the explanation that was outlined in Sec. VI of the relativity of simultaneity in terms of light delays. It uses the aberration formula, which may be deduced from direct visual observations within Real Time Relativity. Along the way we also deduce time dilation and length contraction.

We will refer to Fig. 5, which shows schematic diagrams of the scenario shown in Fig. 4. At event Click both Alice and Bob take photographs of the clocks. We choose Click to be the coincident origins of Alice's and Bob's rest frames, which we assume to be in the standard configuration with relative velocity $v$. Therefore Click occurs at times $t_{A}=t_{B}$ $=0$. In the notation of Sec. III Alice's frame is the camera frame and Bob's frame is the world frame.

Figure 5(a) shows the light paths taken from the clocks $C_{1}$ and $C_{2}$ to Alice, for whom they are moving from right to left with speed $v$. She looks perpendicular to the direction of relative motion to see them at $\theta_{A}=\pi / 2$ and infers that that was their direction when they emitted the light she images. Let the perpendicular distance to clock $C_{1}$ be $d$, and the distance between the clocks in Alice's frame be $L_{A}$. Due to the light propagation delay, the time on the photograph of clock $C_{1}$ will be that it read at time $t_{A}=-d / c$. The path length difference between the paths from clocks $C_{2}$ and $C_{1}$ is

$$
\Delta d_{A}=\sqrt{d^{2}+L_{A}^{2}}-d \approx L_{A}^{2} /(2 d),
$$

where we have assumed $L_{A} \ll d_{A}$ and expanded the square root to first order. The corresponding light propagation time difference can be made arbitrarily small by making $L_{A}$ a sufficiently small fraction of $d$. $^{49}$

Figure 5(b) shows the light paths taken from the clocks to Bob, who is at rest relative to them. He looks back at the angle $\theta_{B}$ to photograph them. Let the distance to clock $C_{1}$ be $d_{B}$. Because lengths perpendicular to the relative motion are invariant, $d_{B}$ is found in terms of $d$ from

$$
d=d_{B} \sin \left(\pi-\theta_{B}\right)=d_{B} \sin \theta_{B} \Rightarrow d_{B}=\gamma d,
$$

where we used the aberration relation Eq. (3), with $\theta_{A}$ $=\pi / 2$ (that is, $\left.\theta_{c}=\pi / 2\right)$, to find $\sin \theta_{B}=\gamma^{-1}$. Due to the light propagation time from $C_{1}$ to Bob, the time on $C_{1}$ 's photograph will be what it read at time $t_{B}=-d_{B} / c=-\gamma d / c$. This time differs from the time deduced by Alice by the time dilation factor $\gamma$. Thus we obtain time dilation from aberration.

However, the focus here is on the relativity of simultaneity. The path length difference $\Delta d_{B}$ between the paths from clocks $C_{1}$ and $C_{2}$ may be approximated by a method familiar from diffraction theory. We drop a perpendicular to $C_{2}$ from the line between clock $C_{1}$ and Bob. The distance along this line from the perpendicular to $C_{1}$ is the approximate path length difference. If we use the corresponding right-angle triangle with hypotenuse $L_{B}$ and angle $\pi-\theta_{B}$, we have

$$
\Delta d_{B}=L_{B} \cos \left(\pi-\theta_{B}\right)=-L_{B} \cos \theta_{B}=L_{B}(v / c),
$$

where we again used Eq. (3a), with $\theta_{A}=\pi / 2$ (that is, $\theta_{c}$ $=\pi / 2$ ), to find $\cos \theta_{B}=-v / c$. The corresponding light propagation time difference, $\Delta t_{B}=L_{B} v / c^{2}$, is the time difference between the clocks in Bob's photograph. However, it is also the time difference between the clocks in Alice's photograph, because both images are made from the same group of photons; those present at event Click.

Length contraction follows from the observation that the length of the perpendicular dropped to $C_{2}$ is $L_{B} \sin \left(\pi-\theta_{B}\right)$ $=L_{B} \gamma^{-1}$, and that therefore the angle subtended by $L_{B}$ to Bob is $d \theta_{w}=L_{B} \gamma^{-1} / d_{B}=L_{B} \gamma^{-2} / d$. Then using Eq. (5), $d \theta_{c}=\gamma d \theta_{w}$ $=L_{B} \gamma^{-1} / d$. Because $d$ is the perpendicular distance from the camera to the clocks, the distance between the clocks in the camera frame is $L_{A}=L_{B} \gamma^{-1}$, which is length contraction.

We can find the time difference in terms of Alice's quantities by expressing the previous result in the form $L_{B}=\gamma L_{A}$,

$$
\Delta t_{B}=\left(\gamma L_{A}\right)\left(v / c^{2}\right)=\gamma\left(L_{A} v / c^{2}\right),
$$

which is the term responsible for the relativity of simultaneity in the inverse Lorentz transformation,

$$
\Delta t_{B}=\gamma\left(\Delta t_{A}+\Delta x_{A} v / c^{2}\right) .
$$

Thus we have shown how the relativity of simultaneity can be understood in terms of light propagation delays and be deduced from direct observations of clocks.

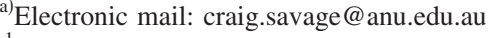

${ }^{1}$ The Real Time Relativity web site contains information about the program and a downloadable installer: /www.anu.edu.au/Physics/Savage/ RTR $\rangle$.

${ }^{2}$ See EPAPS Document No. E-AJPIAS-75-004708 for the simulation software, accompanying manual and the laboratory manual. This document can be reached through a direct link in the online article's HTML reference section or via the EPAPS homepage (http://www.aip.org/pubservs/ epaps.html).

${ }^{3}$ R. E. Scherr, P. S. Shaffer, and S. Vokos, "Student understanding of time in special relativity: Simultaneity and reference frames," Am. J. Phys. 69, S24-S35 (2001).

${ }^{4}$ R. E. Scherr, P. S. Shaffer, and S. Vokos, "The challenge of changing deeply held student beliefs about the relativity of simultaneity," Am. J. Phys. 70, 1238-1248 (2002).

${ }^{5}$ R. E. Scherr, "An investigation of student understanding of basic concepts in special relativity," Ph.D. dissertation, Department of Physics, University of Washington, 2001 (unpublished), (www.physics.umd.edu/ perg/papers/scherr/index.html $>$.

${ }^{6}$ A. Einstein, "Zur elektrodynamik bewegter Körper," Ann. Phys. 17, 891-921 (1905). Reprinted in English translation in J. Stachel, Einstein's
} 
Miraculous Year (Princeton U.P., Princeton, 1998).

${ }^{7}$ A. Komar, "Foundations of special relativity and the shape of the big dipper," Am. J. Phys. 33, 1024-1027 (1965).

${ }^{8}$ A. Peres, "Relativistic telemetry," Am. J. Phys. 55, 516-519 (1987).

${ }^{9}$ H. Blatter and T. Greber, "Aberration and Doppler shift: An uncommon way to relativity," Am. J. Phys. 56, 333-338 (1988).

${ }^{10}$ G. D. Scott and M. R. Viner, "The geometrical appearance of large objects moving at relativistic speeds," Am. J. Phys. 33, 534-536 (1965).

${ }^{11}$ R. R. Hake, "Interactive-engagement versus traditional methods: A sixthousand-student survey of mechanics test data for introductory physics courses," Am. J. Phys. 67, 755-767 (1999).

${ }^{12}$ L. C. McDermott and E. F. Redish, "Resource Letter: PER-1: Physics Education Research,” Am. J. Phys. 66, 64-74 (1998).

${ }^{13}$ L. C. McDermott, "Oersted medal Lecture 2001: Physics education research-the key to student learning," Am. J. Phys. 69, 1127-1137 (2001).

${ }^{14}$ E. Mazur, Peer Instruction: A User's Manual (Prentice Hall, Englewood Cliffs, NJ, 1997).

${ }^{15}$ W. K. Adams, S. Reid, R. LeMaster, S. B. McKagan, K. K. Perkins, and C. E. Wieman, "A study of educational simulations, Parts I and II," J. Interactive Learning Research, in press (2007).

${ }^{16}$ C. Wieman and K. Perkins, "A powerful tool for teaching science," Nat. Phys. 2, 290-292 (2006).

${ }^{17}$ S. Yeo, R. Loss, M. Zadnik, A. Harrison, and D. Treagust, "What do students really learn from interactive multimedia? A physics case study," Am. J. Phys. 72, 1351-1358 (2004).

${ }^{18}$ R. N. Steinberg, "Computers in teaching science: To simulate or not to simulate," Am. J. Phys. 68, S37-S41 (2000).

${ }^{19}$ A. Van Heuvelen, "Learning to think like a physicist: A review of research based instructional strategies," Am. J. Phys. 59, 891-897 (1991)

${ }^{20}$ E. F. Taylor, "Space-time software: computer graphics utilities in special relativity," Am. J. Phys. 57, 508-514 (1989). Spacetime software available from 〈www.eftaylor.com/special.html .

${ }^{21}$ Through Einstein's Eyes, a multimedia web site about relativistic optics. Available on CD from the authors, and online at /www.anu.edu.au/ Physics/Savage/TEE $>$.

${ }^{22}$ N. D. Mermin, It's About Time (Princeton U.P., Princeton, 2005).

${ }^{23}$ R. Penrose, "The apparent shape of a relativistically moving sphere," Proc. Cambridge Philos. Soc. 55, 137-139 (1959).

${ }^{24}$ J. Terrell, "Invisibility of the Lorentz contraction," Phys. Rev. 116, 10411045 (1959)

${ }^{25}$ V. F. Weisskopf, "The visual appearance of rapidly moving objects," Phys. Today 13(9), 24-27 (1960).

${ }^{26}$ W. Rindler, Relativity: Special, General, and Cosmological (Oxford U.P., Oxford, 2006).

${ }^{27}$ The inverse Lorentz transformations of the four-frequency are obtained from Eq. (2) by swapping the $w$ and $c$ labels and changing the sign of $v$.

${ }^{28}$ J. M. McKinley and P. Doherty, "Relativistic transformations of light power," Am. J. Phys. 47, 602-605 (1979).

${ }^{29}$ J. M. McKinley, "In search of the starbow: The appearance of the starfield from a relativistic spaceship," Am. J. Phys. 47, 309-316 (1979).

${ }^{30}$ Men-Chou Chang, Feipei Lai and Wei-Chao Chen, "Image shading taking into account relativistic effects," ACM Trans. Graphics 15, 265 (1996)
${ }^{31}$ T. Müller, "Visual appearance of a Morris-Thorne-wormhole," Am. J. Phys. 72, 1045-1050 (2004).

${ }^{32}$ W. Gekelman, J. Maggs, and Lingyu Xu, "Real-time relativity," Comput. Phys. 5, 372-385 (1991).

${ }^{33}$ Ping-Kang Hsiung, R. H. Thibadeau, and M. Wu, "T-buffer: Fast visualization of relativistic effects in space-time," Comput. Graph. 24, 83-88 (1990).

${ }^{34}$ J. D. Owens et al., "A survey of general-purpose computation on graphics hardware,” Comput. Graph. Forum 26, 80-113 (2007); 〈graphics.idav.ucdavis.edu/publications〉.

${ }^{35}$ D. Weiskopf, "Visualization of four-dimensional spacetimes," Ph.D. dissertation, University of Tübingen, 2001 (unpublished); 〈www.vis.unistuttgart.de/relativity/reading $\rangle$.

${ }^{36} \mathrm{M}$. Bochers, "Interactive and stereoscopic visualization in special relativity," Ph.D. dissertation, University of Tübingen, 2005 (unpublished); 〈w210.ub.uni-tuebingen.de/dbt/volltexte/2005/1891〉.

${ }^{37}$ U. Kraus and C. Zahn, "Relativity visualized: Space time travel,"〈www.spacetimetravel.org $\rangle$.

${ }^{38}$ D. Weiskopf et al., "Explanatory and illustrative visualization of special and general relativity," IEEE Trans. Vis. Comput. Graph. 12, 522-534 (2006).

${ }^{39}$ T. Greber and H. Blatter, "Aberration and Doppler shift: The cosmic background radiation and its rest frame," Am. J. Phys. 58, 942-945 (1990).

${ }^{40}$ U. Kraus, "Brightness and color of rapidly moving objects: The visual appearance of a large sphere revisited," Am. J. Phys. 68, 56-60 (2000).

${ }^{41}$ Computer system requirements for running Real Time Relativity are Microsoft Windows 98/Me/2000/XP with DirectX 9, Resolution 1024 $\times 768$ or greater, color Depth 24 bit or greater, and a DirectX 9 compatible video card running PS2.0 or greater, such as nVidia GeForce FX Series, 6xxx Series or 7xxx Series or ATI Radeon 9500-9800 Series, or X Series. It generally will not run on motherboard integrated graphics systems.

${ }^{42}$ J. Andrews and N. Baker, "Xbox 360 system architecture," IEEE Micro 26, 25-37 (2006).

${ }^{43}$ Folding@home project web site 〈folding.stanford.edu〉.

${ }^{44}$ The lectures and tutorials were 50 minutes each. There were 74 students in the class.

${ }^{45}$ The Real Time Relativity laboratory manual is available at EPAPS (Ref. 2) and the Real Time Relativity web site (Ref. 1).

${ }^{46}$ G. Gamow, Mr Tompkins in Paperback (Cambridge U.P., Cambridge, 1965). The figures do not show what would actually be seen, which was not known at the time the story was written.

${ }^{47}$ Although the Sun and Earth are the correct size (the Sun's diameter is 4.7 light-seconds) the distance between them is not to scale.

${ }^{48}$ The best way to experience this sensation is to run the simulation. A movie of the sequence discussed in the text is available from Ref. 2 or 1.

${ }^{49}$ If the perpendicular distance to the clocks is sufficiently large compared to the distance between the clocks, this approximation is as good as required. It is only within this approximation that pure length contraction and relativity of simultaneity are observed.

${ }^{50}$ The quotations are taken from the post-laboratory evaluations completed by students. 